\title{
Le risorse della Cardiologia Riabilitativa
}

\author{
Sabino Scardi
}

RIASSUNTO: Le risorse della Cardiologia Riabilitativa. S. Scardi.

Le Unità Operative di riabilitazione sono al momento attuale le strutture che possiedono le migliori potenzialità per garantire la continuità della cura dei cardiopatici nel territorio, perché il cardiologo riabilitatore ha la cultura del cronico. Tuttavia l'esiguità delle strutture, le scarse potenzialità, l'insufficienza del personale, il numero crescente di utenti che necessitano di continuità assistenziale (ad esempio anziani) limitano la potenzialità dell'intervento riabilitativo a lungo termine.
In alternativa la costituzione di organici Centri Cardiologici Territoriali, dotati di personale e attrezzature adeguati, in stretta collaborazione con l'ospedale e il medico di medicina generale, sarebbero in grado di raccordare la cura acuta con quella a lungo termine, avviando programmi di continuità assistenziale per assicurare il follow-up dei malati.

Keywords: cardiac rehabilitation, continuity of care, quality of health care.

Monaldi Arch Chest Dis 2006; 66: 129-132.

Centro Cardiovascolare, Trieste.

In questi ultimi anni c'è stata un'evoluzione della domanda di salute in quanto sono emerse le nuove esigenze legate alla cronicità. Se il cronico non può essere curato in un ospedale per acuti, ciò richiederà nuovi modelli organizzativi ed assistenziali nel territorio. Pertanto, la sanità pubblica deve adeguare il sistema di cura ai nuovi bisogni legati alla cronicità; in questo contesto, devono rinnovarsi anche gli interessi della cardiologia [1-2]. Sappiamo che i trattamenti attuali efficaci migliorano la prognosi, soprattutto nei pazienti ad alto rischio [3]; il rischio diventa però più elevato nei sopravvissuti; la progressione della malattia determina un ulteriore aumento del rischio con la richiesta di nuovi e ulteriori trattamenti, in questo modo si instaura quella spirale che si conclude solo con il decesso del paziente (fig. 1).

Le malattie cardiache sono complesse da gestire, sia trasversalmente sia longitudinalmente, perché non sono stabili, e richiedono perciò una periodica rivalutazione da parte di personale esperto [4].

Nonostante (a capo) l'utilità di alcune terapie dimostrata dai trial, il loro utilizzo rimane basso nei cardiopatici non seguiti dal cardiologo. Per esempio, nella prevenzione primaria e secondaria della cardiopatia ischemica, il controllo dei fattori di rischio risulta inadeguato nella pratica clinica [5-6]. Le cause sono diverse:

- l'insufficiente azione dei medici: il cardiologo, più impegnato nella "cura", demanda al medico di medicina generale, il quale, spesso oberato da problemi burocratici e di assistenza, dimentica la prevenzione;

- scarso follow-up dei fattori di rischio non modificabili con i farmaci (fumo ed attività fisica);

- pazienti poco motivati a modificare uno stile di vita oramai radicato.

Esistono, poi, una serie di errori nella gestione del cardiopatico cronico:

- scarsa attenzione a rallentare la progressione della malattia;

- non riconoscimento della progressione della malattia;
- uso improprio o inadeguato di farmaci;

- uso di farmaci potenzialmente dannosi;

- scarsa attenzione alle comorbilità.

Se un paziente ischemico dal territorio acutamente arriva in ospedale, in unità coronarica, va in degenza, va in riabilitazione e poi in post-degenza, e quando torna nel territorio deve trovare la cosiddetta continuità assistenziale (fig. 2) [7]. C'è una grande confusione al riguardo; vengono usati come sinonimi: la continuità della cura, la coordinazione, il piano di dimissione, il case management, l'integrazione dei servizi, il semless care, il patient/clientoriented, le cure condivise (shared care), e l'integrated care. La continuità assistenziale è un progetto unitario di "care", cioè di prendersi cura e di presa in carico delle persone che deve essere personalizzato e multidisciplinare, con il coinvolgimento, non solo del personale medico (medici di medicina generale e specialisti), ma anche di quello non medico [4]. Attualmente un cardiopatico dimesso dall'ospedale ha a sua disposizione quattro opzioni: il suo medico di medicina generale, ed è giusto che lo abbia, lo specialista ambulatoriale convenzionato, l'ambulatorio ospedaliero e il cardiologo personale. Ognuna di queste soluzioni assicura non una cura, ma una semplice consulenza incompleta spesso nemmeno somministrata al momento del bisogno reale. La continuità assistenziale, invece, deve passare per un percorso di conoscenza fra i diversi attori abilitati a seguire il caso: il medico di medicina generale, lo specialista e l'infermiere e soprattutto deve realizzare un progetto di salute preordinato, articolato, condiviso e di presa in carico da parte dei servizi sanitari per assicurare al paziente la continuità di un percorso coerente.

La riabilitazione cardiovascolare è sicuramente un mezzo primario per assicurare una continuità assistenziale. La struttura riabilitativa ambulatoriale, infatti, in questo contesto, ha avuto un ruolo guida perché ha anticipato di molti anni la cultura della cura del cardiopatico cronico [8].

La riabilitazione cardiovascolare è un modello standard per il trattamento globale del cardiopatico 


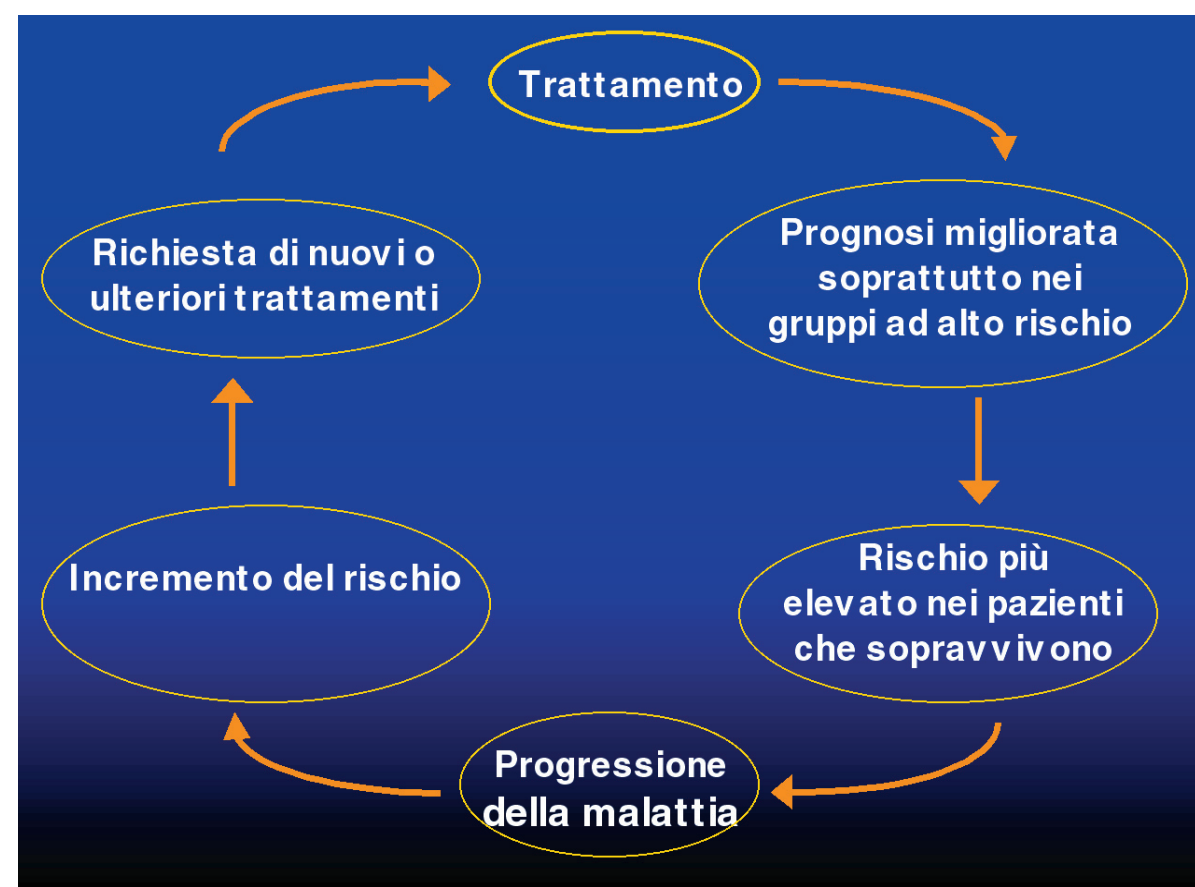

Figura 1. - La spirale del rischio.

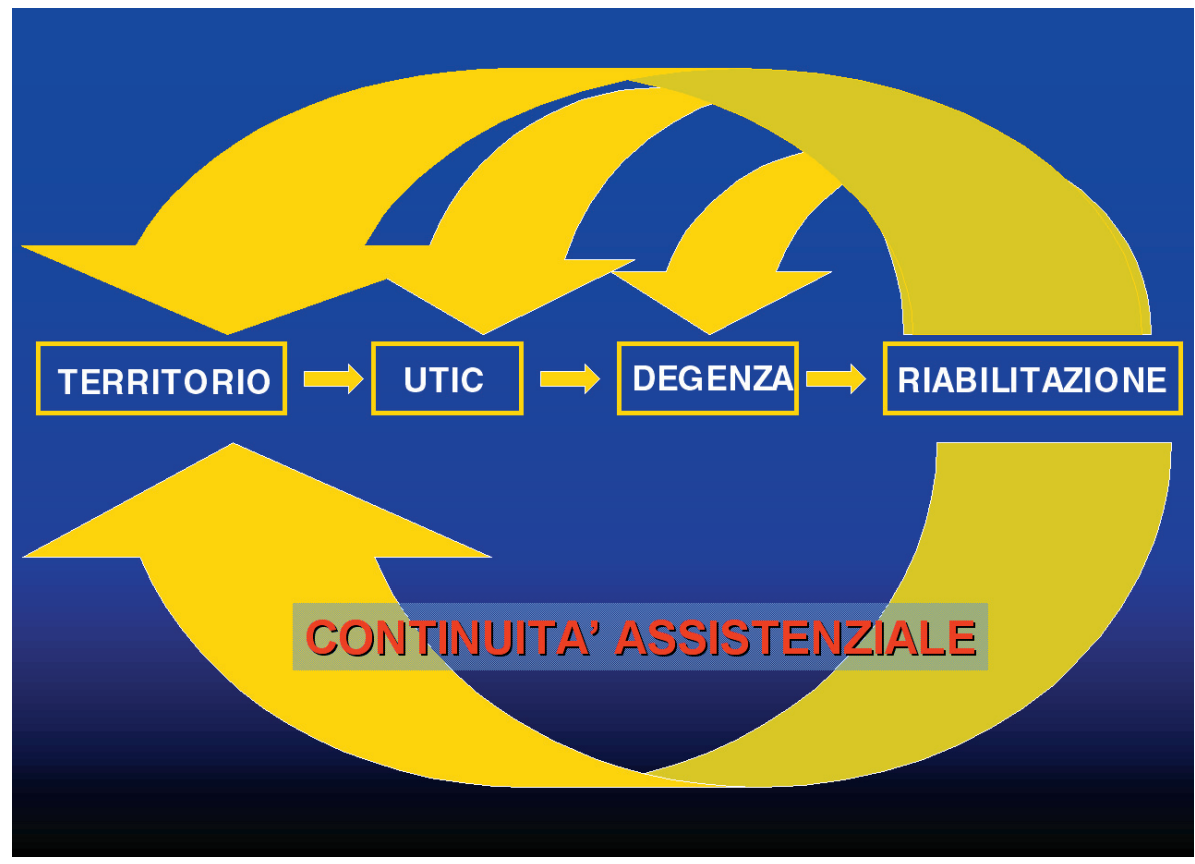

Figura 2. - La continuità assistenziale ospedale-territorio.

[9] e rappresenta una componente importante per un programma assistenziale a lungo termine [10]. La riabilitazione è importante perché fa prevenzione secondaria, stratificazione prognostica, follow-up clinico e strumentale e ottimizza la terapia, assicurando in questo modo non solo un prolungamento della quantità della vita, ma soprattutto una migliore qualità [11-12].

Il cardiologo riabilitatore è preparato a seguire il cardiopatico nel tempo perché ha la cultura del cronico. Tuttavia, l'esiguità numerica delle strutture, le differenti potenzialità fra strutture con degenza e ambulatoriali, l'insufficienza del personale, il numero crescente di pazienti che necessitano di continuità assistenziale (vedi l'anziano) hanno limitato la potenzialità dell'intervento riabilitativo a lungo termi- ne, anche se, laddove è stato realizzato, i risultati ottenuti sono stati soddisfacenti [13].

\section{Come garantire}

la continuità assistenziale dall'ospedale al territorio?

Sicuramente lo specialista rappresenta il terzo vertice di un triangolo che fa capo ad un medico di medicina generale e al paziente e ha un ruolo chiave nella gestione delle malattie croniche perché assicura un supporto decisionale e coordina le azioni fra l'infermiere specializzato nella cura cronica e il medico di medicina generale [4]. La cura delle malattie cardiovascolari croniche nel territorio è attualmente relativamente costosa, ma poco efficace per raggiungere obiettivi di salute, in quanto gravata da un circolo di disease management che comprende visite di medicina generale, del cardiologo ambulatoriale, frequenti accessi in pronto soccorso, frequenti riospedalizzazioni. Pertanto, i brillanti risultati delle cure acute possono risultare poco utili per l'insufficiente successiva gestione territoriale [14]. E necessario perciò migliorare la qualità della cura programmando, accanto alla rete ospedaliera, la rete integrata ospedale-territorio che assicuri, insieme al medico di medicina generale, un programma di assistenza territoriale che, se efficace, può ridurre il ricorso al ricovero improprio, identificare eventi minori prodromici di eventi maggiori, prevenire aggravamenti con interventi diagnostico-terapeutici precoci e tempestivi, migliorare la quantità e la qualità della vita dei cardiopatici, assicurando un risultato assistenziale, e non solo medico.

Il problema della continuità assistenziale è tuttavia molto complesso e richiede da parte delle associazioni culturali, come questa, e del legislatore un ripensamento ed uno sforzo diretti ad assicurare una più razionale organizzazione dell'assistenza ambulatoriale sia ospedaliera sia territoriale [14]. Il medico di medicina generale, a cui spetta ordinare le prestazioni e la titolarità del budget, è l'elemento fondamentale delle cure primarie perché deve coordinare la continuità assistenziale. La presa in carica di pazienti complessi, tuttavia, richiede professionalità specialistica e multidisciplinare per 
patologie che un medico di medicina generale non sempre da solo può affrontare. Una vera continuità assistenziale si potrà realizzare solo se, al medico di medicina generale, si affiancherà nel territorio, personale medico ed infermieristico realmente esperto in cardiologia, o in altre discipline affini. Il territorio ha, tuttavia, un vuoto cardiologico, ha una scarsa dotazione strumentale, è privo di un organico strutturato, limitato spesso alla presenza del singolo specialista, è scollegato dall'ospedale. Pertanto, l'attitudine alla cura del cardiopatico cronico deve provvedere alla creazione di una rete operativa che raccordi la cura acuta con quella a lungo termine, assicuri il follow-up della rivalutazione dei malati, avvii i programmi di continuità assistenziale.

\section{Quali ipotesi organizzative per assicurare questa continuità assistenziale?}

Contrariamente alle tradizionali strutture che prevedono l'intervento crisi, una strategia vincente deve favorire l'aggregazione fra i servizi stabilendo percorsi condivisi e idonei, l'appropriatezza dei ricoveri e delle cure, l'utilizzazione più razionale delle risorse. Ciò richiede un rivoluzionario cambiamento dell'organizzazione specialistica territoriale, modificando il sistema di cura, ridisegnando i servizi specialistici, ripensando il ruolo di alcuni cardiologi ospedalieri. Gli ospedali di rete, per esempio, devono essere totalmente reintegrati nei servizi ambulatoriali specialistici territoriali, dopo un adeguamento dell'organico e della strumentazione. Negli ospedali di più grosse dimensioni, invece, è necessario stabilire un nuovo rapporto fra strutture ospedaliere, strutture specialistiche ambulatoriali, medico di medicina generale, che devono svolgere attività integrata. Pertanto, l'ipotesi organizzativa è quella di:

- creare centri cardiologici territoriali con proprio organico strutturato, nel quale includere gli attuali cardiologi ambulatoriali coordinati da un responsabile;

- creare dipartimenti interaziendali ospedaleterritorio;

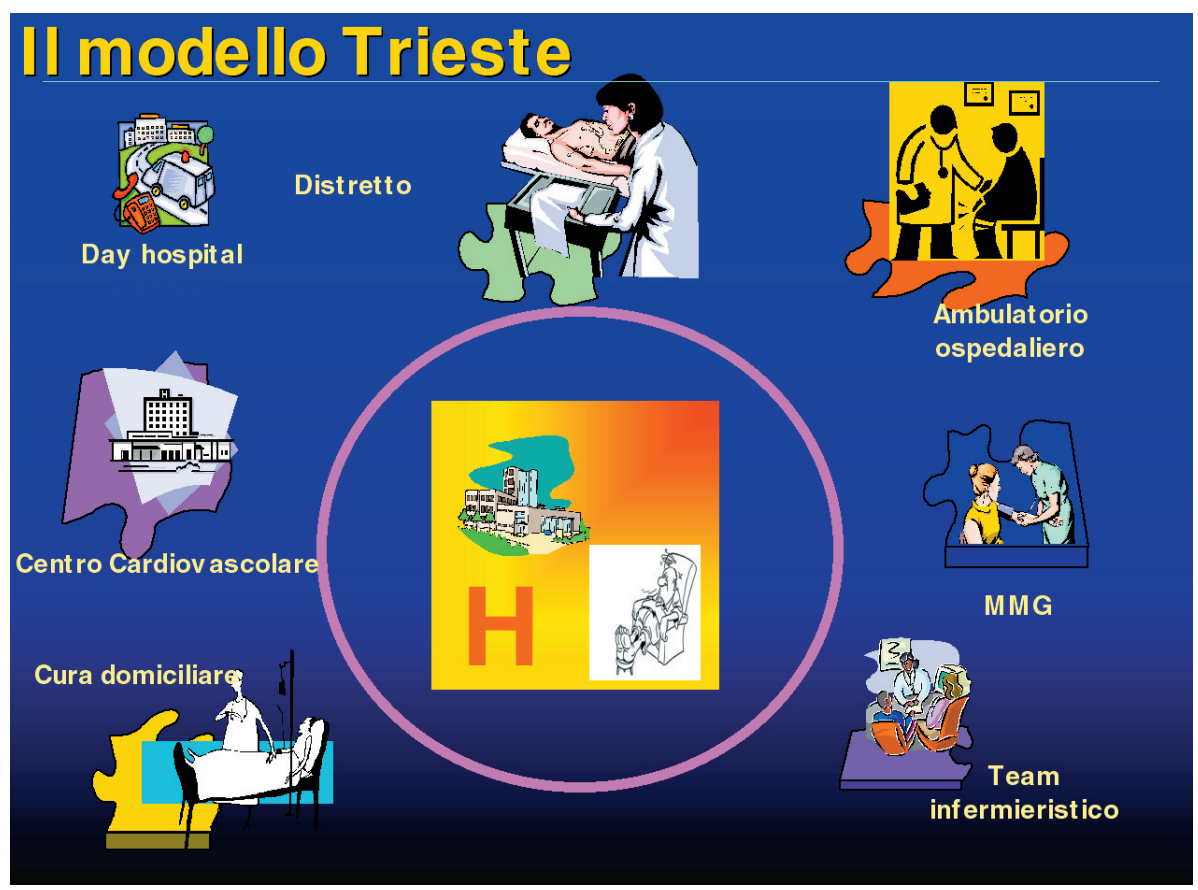

Figura 3. - I collegamenti funzionali del Centro Cardiologico territoriale.

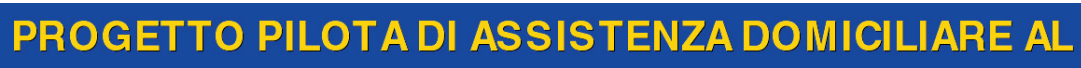
PAZIENTE CON SCOMPENSO CARDIACO a Trieste

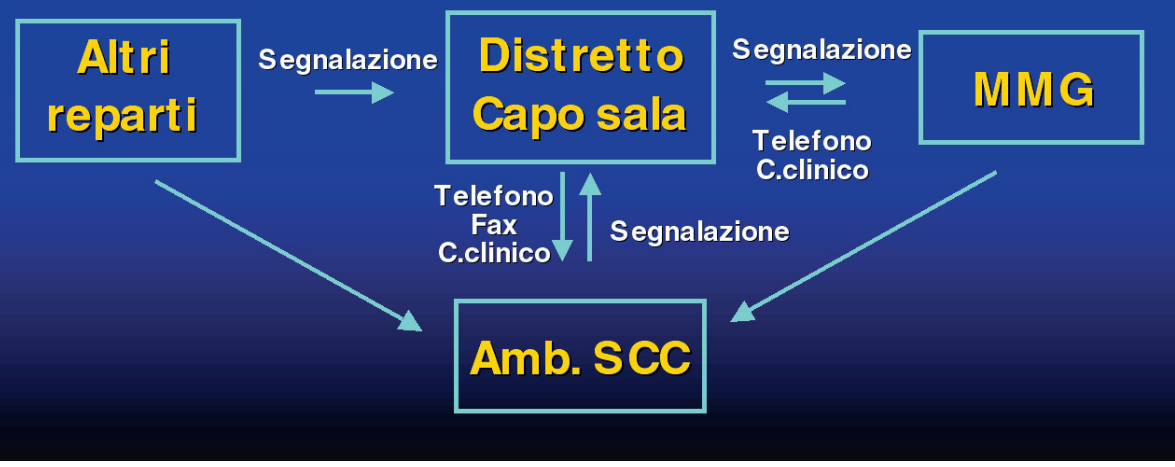

Figura 4. - I collegamenti nel progetto Trieste Heart Failure. dalieri;

- incrementare l'organico dei cardiologi ospe-

- alcuni cardiologi clinici ospedalieri, ed in particolare quelli più anziani, ruoteranno nel territorio per diffondere cultura dell'ospedale nel territorio e cultura del territorio nell'ospedale.

Il modello attuale è tutto basato sull'ospedale, il telemonitoraggio, il day hospital, l'ambulatorio cardiologico, l'ambulatorio dello scompenso, mentre vediamo in periferia il medico di medicina generale e lo specialista convenzionato isolati nell'ambulatorio del distretto. Da anni abbiamo attuato a Trieste un nuovo modello, in cui, accanto alla struttura classica dell'ospedale, della cardiologia ospedaliera e degli ambulatori specialistici, abbiamo creato il centro cardiologico territoriale, in stretta collabora- 
zione con l'ospedale e con il medico di medicina generale [15]. I collegamenti funzionali di questa struttura sono con la divisione di cardiologia e di cardiochirurgia mediante il Dipartimento Interaziendale, con i distretti e con i medici di medicina generale (fig. 3). Il personale è costituito da un medico dirigente di secondo livello, otto dirigenti di primo livello, nove infermieri e una caposala. I compiti sono di tipo epidemiologici preventivi, diagnostico-terapeutici, riabilitazione ambulatoriale a lungo termine, continuità assistenziale compresa quella domiciliare. I pazienti che necessitano di continuità assistenziale vengono segnalati al centro cardiologico territoriale da parte della divisione di cardiologia e cardiochirurgia (nessun paziente viene dimesso da noi senza sapere da chi andare dopo), da altre strutture ospedaliere, o dal medico di medicina generale. Questi pazienti vengono inclusi in un database e richiamati alla scadenza mediante lettera o telefono o su richiesta del medico di medicina generale. Nella nostra ipotesi per lo scompenso cardiaco, in funzione da un anno e mezzo, i pazienti delle varie divisioni ospedaliere, compresa la cardiologia, vengono segnalati alla caposala del distretto, e questa a sua volta collega il centro cardiologico territoriale con i reparti o con il medico di medicina generale o con l'ambulatorio dello scompenso (fig. 4). Tutto questo assicura al paziente la cosiddetta continuità assistenziale.

\section{Conclusioni}

In conclusione, la cronicità è un nuovo scenario con cui la medicina in generale e la cardiologia in particolare devono confrontarsi per sviluppare risposte assistenziali applicabili, efficaci e sostenibili per assicurare la continuità della cura. Il nostro modello organizzativo non può essere imposto con decreto ma con una discussione a livello nazionale, a livello regionale, e a livello locale. La proposta operativa suggerita, al di là dei facili slogan, deve essere discussa seriamente, eliminando alcune barriere che ostacolano il raggiungimento di questo obiettivo.

\section{Bibliografia}

1. Wagner EH. Meting the needs of chronically ill people. Br Med J 2001; 323: 945-946.

2. Freer SD. Whither continuity of care? New Engl J Med 1999; 341: 850-852.

3. McGlynn EA, Asch SM, Adams J, et al. The quality of health care delivered to adults in the United States. New Engl J Med 2003; 38, 2635-2645.

4. Gask L. Role of specialists in common chronic diseases. Br Med J 2005; 330: 651-653.

5. Gislason GH, Rasmussen JN, Abildstrom SZ, et al. Longterm compliance with beta-blockers, angiotensin-converting enzyme inhibitors, and statins after acute myocardial infarction. Eur Heart J 2006; 27: 1153-1158.

6. Reimer WS, De Swart E, D Bacquer D, et al. Smoking behaviour in european patients with estalished coronary heart disease. Eur Heart J 2005; 2: 35-41.

7. Scardi S. La dimissione dei pazienti con infarto miocardio: evoluzione delle strategie. G Riab 1998; 14: 1-5.

8. Scardi S, Ignone G. Valutazione funzionale e riabilitazione del cardiopatico. UTET, Torino, 1993.

9. Scardi S. Riabilitazione: dove, come, quando? In Temi di Cardiologia Pozzi, Roma 1982.

10. Cobelli F, Giordano A, Sala L, Scardi S. Riabilitazione cardiologica: linee guida per una corretta strategia operativa GIVFRC, Milano 1992.

11. Swedberg K, Clelend J, Dargie H, et al. Guidelines for the diagnosis and treatment of chronic heart failure: executive summary (update 2005). EHJ 2005; 26, 1115-1140.

12. Ades PA. Cardiac rehabilitation and secondary prevention of coronary artery disease. New Engl J Med 2001; 345: 892-902.

13. Scardi S, Mazzone C. Riabilitazione cardiovascolare: valore aggiunto per la prevenzione secondaria? Monadi Arch Chest Dis 2003; 60: 1-6.

14. Haggerty JL, Reid RJ, Freeman GK, et al. Continuity of care: a multidisciplinary review. Br Med J 2003; 327: 1219-1221.

15. Scardi S. Il collegamento fra le strutture cardiologiche ospedaliere ed il territorio. Atti IX Congresso Nazionale ANMCO Firenze 1978: 21-30. 